Article

\title{
Glycyrrhizin Alleviates Neuroinflammation and Memory Deficit Induced by Systemic Lipopolysaccharide Treatment in Mice
}

\author{
Jeong-Ho Song, Ju-Won Lee, Beomsoo Shim, Chang-Yeol Lee, Sooyong Choi, Chulhun Kang, \\ Nak-Won Sohn and Jung-Won Shin * \\ Department of East-West Medical Science, Graduate School of East-West Medical Science, \\ Kyung Hee University, Yongin 446-701, Korea; E-Mails: beginbegun@hanmail.net (J.-H.S.); \\ won8502@hanmail.net (J.-W.L.); bom-su@hanmail.net (B.S.); cylee81@hanmail.net (C.-Y.L.); \\ csyomd@hanmail.net (S.C.); kangch@khu.ac.kr (C.K.); sohnnw@khu.ac.kr (N.-W.S.) \\ * Author to whom correspondence should be addressed; E-Mail: shinarago@khu.ac.kr; \\ Tel.: +82-312-012-747; Fax: +82-312-046-832.
}

Received: 5 November 2013; in revised form: 9 December 2013 / Accepted: 10 December 2013 / Published: 17 December 2013

\begin{abstract}
The present study investigated the effects of glycyrrhizin (GRZ) on neuroinflammation and memory deficit in systemic lipopolysaccharide (LPS)-treated C57BL/6 mice. Varying doses of GRZ was orally administered (10, 30, or $50 \mathrm{mg} / \mathrm{kg}$ ) once a day for 3 days before the LPS ( $3 \mathrm{mg} / \mathrm{kg}$ ) injection. At $24 \mathrm{~h}$ after the LPS injection, GRZ significantly reduced TNF- $\alpha$ and IL- $1 \beta \mathrm{mRNA}$ at doses of 30 and $50 \mathrm{mg} / \mathrm{kg}$. COX-2 and iNOS protein expressions were significantly reduced by GRZ at doses of 30 and $50 \mathrm{mg} / \mathrm{kg}$. In the Morris water maze test, GRZ (30 mg/kg) significantly prolonged the swimming time spent in the target and peri-target zones. GRZ also significantly increased the target heading and memory score numbers. In the hippocampal tissue, GRZ significantly reduced the up-regulated Ibal protein expression and the average cell size of Iba1-expressing microglia induced by LPS. The results indicate that GRZ ameliorated the memory deficit induced by systemic LPS treatment and the effect of GRZ was found to be mediated through the inhibition of pro-inflammatory mediators and microglial activation in the brain tissue. This study supports that GRZ may be a putative therapeutic drug on neurodegenerative diseases associated with cognitive deficits and neuroinflammation such as Alzheimer's disease.
\end{abstract}

Keywords: glycyrrhizin; neuroinflammation; memory deficit; microglial activation 


\section{Introduction}

Glycyrrhizin (GRZ), a triterpenoid saponin compound, is the main constituent of Glycyrrhiza glabra and is composed of a molecule of glycyrrhetinic acid and two molecules of glucuronic acid [1,2]. GRZ given orally is absorbed into the bloodstream as $18 \beta$-glycyrrhetinic acid (GA), then GA reaches the brain through the brain-blood barrier [3]. GRZ has been reported to have various pharmacological effects, including anti-inflammatory and neuroprotective effects. Its anti-inflammatory outcome is by suppressing the expression of pro-inflammatory cytokine genes through the inhibition of nuclear factor- $\kappa \mathrm{B}(\mathrm{NF}-\kappa \mathrm{B})$ and phosphoinositide-3-kinase (PI3K) activity [4], and by attenuating excessive nitric oxide (NO) and reactive oxygen species (ROS) production [4,5]. GRZ also suppresses inducible nitric oxide synthase (iNOS) expression and reduces prostaglandin E2 (PGE2) release through the inhibition of cyclooxygenase-2 (COX-2) [6,7]. It is suggested that the inhibitory action of GRZ on NF- $\mathrm{B}$ and PI3K activities protect neurons from glutamate-induced excitotoxicity and ischemic injury [8,9]. Recently, a line of in vivo studies reported that GRZ exerted neuroprotective effects against cerebral ischemia, intracerebral hemorrhage, and ischemic spinal cord injury via its anti-inflammatory effects [10-13]. These reports suggested that GRZ plays an inhibitory role on high mobility group box 1 (HMGB1) protein. HMGB1 behaves like an early pro-inflammatory cytokine to promote inflammation [14] and serves as a risk factor for memory impairment, neurodegeneration, and progression of neuroinflammation [15]. Moreover, previous reports exhibited that GRZ has spatial memory enhancing effect [1] and ameliorating effect on cognitive impairment induced by beta-amyloid $(\mathrm{A} \beta)$ injection into the hippocampus [16]. The cognitive ameliorating effect of GRZ was supported by its suppressing effect on $A \beta$-induced microglial activation and inflammation in vitro and in vivo [17].

Neuroinflammation, the inflammation associated with the brain, is characterized by the activation of microglia and expression of major inflammatory mediators without typical features of peripheral inflammation such as edema and neutrophil infiltration [18]. Neuroinflammation causes cognitive impairment, even if it is acutely stimulated by immunostimulatory component such as lipopolysaccharide (LPS) [19]; and its chronic state contributes to progression of neurodegenerative diseases including Alzheimer's disease (AD) [20]. Systemic treatment of LPS stimulates the inflammatory responses in the brain through the toll-like receptor-4 mediated signaling pathway [21]. Upon exposure to LPS, microglia are activated and produce pro-inflammatory mediators such as cytokines, chemokines, prostanoids, and reactive oxygen species [22]. Microglia are the primary cellular source of pro-inflammatory cytokines, including tumor necrosis factor- $\alpha$ (TNF- $\alpha$ ) and interleukin (IL)-1 $\beta$, detected in the brain [23]. Pro-inflammatory cytokines disrupt hippocampal neuronal functions such as long-term potentiation (LTP) and working memory consolidation [24,25]. Consequently, LPS induces a complex array of behaviors known as "sickness behaviors" [26] and leads to alterations in central processes involved in learning and memory [19,27]. Therefore, systemic or intraventricular LPS injection into rodents is popularly used as a model for studying the interaction between inflammation, brain functions, and memory deficits [19,24,25,27].

To better understand anti-neuroinflammatory effect of GRZ, the present study investigated its effects on TNF- $\alpha$, IL-1 $\beta$, COX-2, and iNOS expression in the brain tissue; microglial activation in the hippocampus; and on learning and memory deficits induced by systemic LPS treatment in mice. 


\section{Results and Discussion}

\subsection{Effects on TNF- $\alpha$ and IL-1 $\beta$ Expressions in the Brain Tissue of LPS-Treated Mice}

Systemic treatment of LPS stimulates pro-inflammatory cytokines, including TNF- $\alpha$ and IL-1 $\beta$, in the brain. Compared to serum, the brain showed significantly higher levels of TNF- $\alpha$ and IL-1 $\beta, 28 \mathrm{~h}$ post a single systemic injection of LPS [28]. Additionally, mRNA/protein expression of inflammatory mediators in the brain appeared within 4-8 $\mathrm{h}$ and subsided in 1-3 days after a single LPS injection $[29,30]$. In this study, TNF- $\alpha$ and IL- $1 \beta$ mRNA levels were measured $24 \mathrm{~h}$ after LPS $(3 \mathrm{mg} / \mathrm{kg})$ injection. LPS induced robust increases of TNF- $\alpha$ and IL- $1 \beta$ mRNA in the brain tissue compared to the normal group. GRZ treatment significantly reduced TNF- $\alpha$ mRNA level at all doses of 10, 30 and $50 \mathrm{mg} / \mathrm{kg}$ ( $p<0.05$; respectively) and decreased IL-1 $\beta$ mRNA level at doses of 30 and $50 \mathrm{mg} / \mathrm{kg}$ ( $p<0.05$; respectively) compared to the LPS group (Figure 1). In addition, TNF- $\alpha$ expression was observed with immunohistochemistry in the brain tissue of the mice which performed the Morris water maze test. The LPS group showed significant increases of relative density of TNF- $\alpha$ expression in the cerebral cortex $(p<0.001)$ and dentate gyrus (DG) region of the hippocampus $(p<0.001)$ compared to that of the normal group. The LPS+GRZ group $(30 \mathrm{mg} / \mathrm{kg})$ demonstrated significant reductions in relative densities of TNF- $\alpha$ expression in the cerebral cortex $(p<0.05)$ and DG region of the hippocampus $(p<0.01)$ compared to that of the LPS group (Figure 2A,B). The results in this study indicate that GRZ reduced the over-expression of TNF- $\alpha$ and IL-1 $\beta$ induced by systemic LPS treatment in the brain tissue. Previous reports have demonstrated that GRZ effectively suppressed TNF- $\alpha$ and IL-1 $\beta$ expression in ulcerative colitis [31], in post ischemic brain [10], and in spinal cord injuries $[12,13]$. The results suggest that GRZ exerts anti-neuroinflammatory effects.

Figure 1. Effects of GRZ on TNF- $\alpha$ and IL-1 $\beta$ mRNA in the brain tissue. GRZ significantly attenuated the up-regulation of brain TNF- $\alpha$ mRNA at all doses of 10, 30 and $50 \mathrm{mg} / \mathrm{kg}$ (A) IL-1 $\beta$ mRNA was attenuated at 30 and $50 \mathrm{mg} / \mathrm{kg}$ of GRZ; and (B) Data are represented by mean $\pm \operatorname{SEM}\left(\mathrm{n}=6\right.$ in each group; ${ }^{*} p<0.05$ compared to the LPS group).
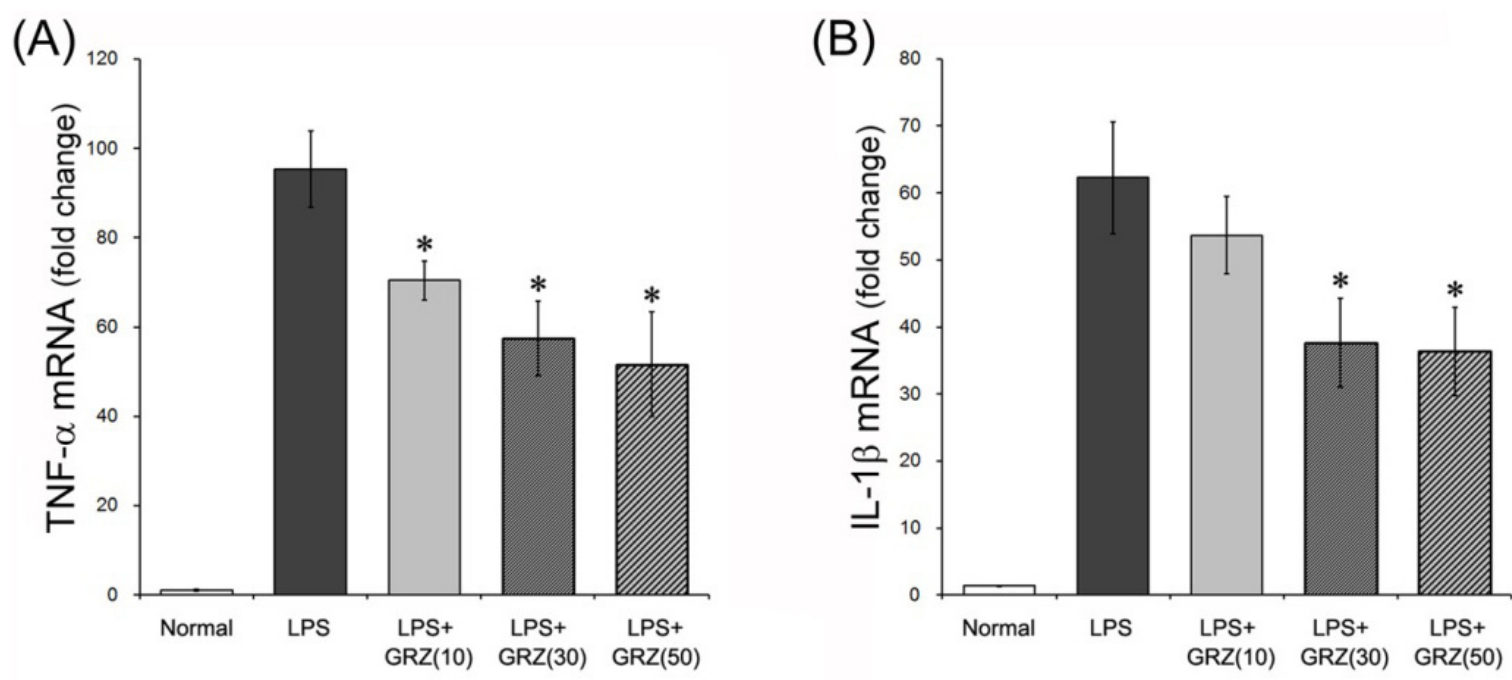
Figure 2. Effect of GRZ on TNF- $\alpha, \mathrm{COX}-2$, and iNOS expression in brain tissue immunohistochemistry. Representative TNF- $\alpha$-, COX-2- and iNOS-expressing cells in the brain tissue of mice which performed the Morris water maze test. Scale bars are $100 \mu \mathrm{m}$ (A,C,E) GRZ (30 mg/kg) significantly attenuated the relative immune-densities of TNF- $\alpha$ (B) COX-2 (D) and iNOS; and (F) in the cerebral cortex or in the dentate gyrus of hippocampus. Data are represented by mean \pm SEM $(n=6$ in each group; $* p<0.05$; $* * p<0.01 ; * * * p<0.001$ compared to the LPS group).
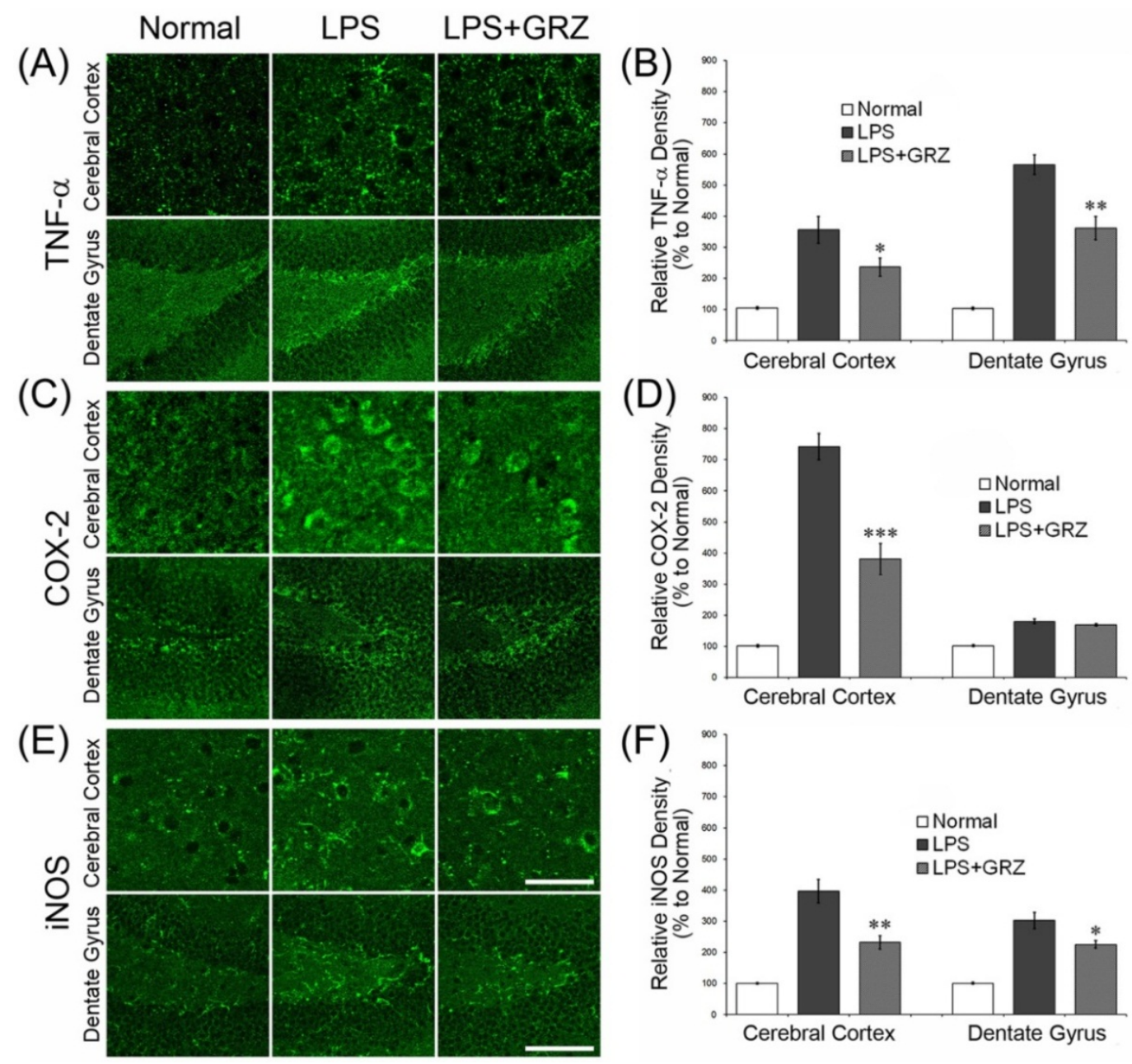

\subsection{Effects on COX-2 and iNOS Expressions in the Brain Tissue of LPS-Treated Mice}

COX-2 plays a central role in the inflammatory cascade by producing prostaglandin in acute and chronic inflammatory conditions [32]. NO is a major pleiotrophic mediator produced by iNOS and reacts with superoxide to form the powerful oxidant peroxynitrite [33]. In this study, COX-2 and iNOS expressions were measured $24 \mathrm{~h}$ post LPS injection using a western blotting method. GRZ treatment significantly reduced the increase of COX-2 expression at all doses of 10,30 and $50 \mathrm{mg} / \mathrm{kg}$ ( $p<0.01, p<0.001, p<0.001$; respectively) and also reduced the increase of iNOS expression at doses of 30 and $50 \mathrm{mg} / \mathrm{kg}$ ( $p<0.05, p<0.01$; respectively) compared to the LPS group (Figure 3 ). In addition, COX-2 and iNOS expressions were observed with immunohistochemistry in the brain tissue of the mice which performed the Morris water maze test. The LPS+GRZ group (30 $\mathrm{mg} / \mathrm{kg})$ demonstrated significant reductions in relative densities of COX-2 and iNOS expression in the cerebral 
cortex and DG region of the hippocampus compared to that of the LPS group (Figure 2C-F). The results support that GRZ exerts anti-neuroinflammatory effect, considering a line of results in previous studies and its effects on TNF- $\alpha$ and IL- $1 \beta$ mRNA in the present study. GRZ has been shown to suppress COX-2 and iNOS expressions in acute lung injury induced by LPS treatment [6]. GRZ also showed an anti-inflammatory effect by attenuating the generation of excessive NO, PGE2, and ROS through the inhibition of NF-אB and PI3K activity in various in vitro and in vivo studies [4-8].

Figure 3. Effects of GRZ on COX-2 and iNOS protein expression in the brain tissue. Representative western blots illustrating differences in the bands of COX-2 and iNOS (A) GRZ significantly attenuated the up-regulation of brain COX-2 expression at all doses of 10,30 and $50 \mathrm{mg} / \mathrm{kg}$; (B) and iNOS expression was attenuated at 30 and $50 \mathrm{mg} / \mathrm{kg}$ of GRZ; and $(\mathbf{C})$ Data are represented by mean \pm SEM $\left(n=6\right.$ in each group; ${ }^{* *} p<0.01$; *** $p<0.001$ compared to the LPS group).

(A)
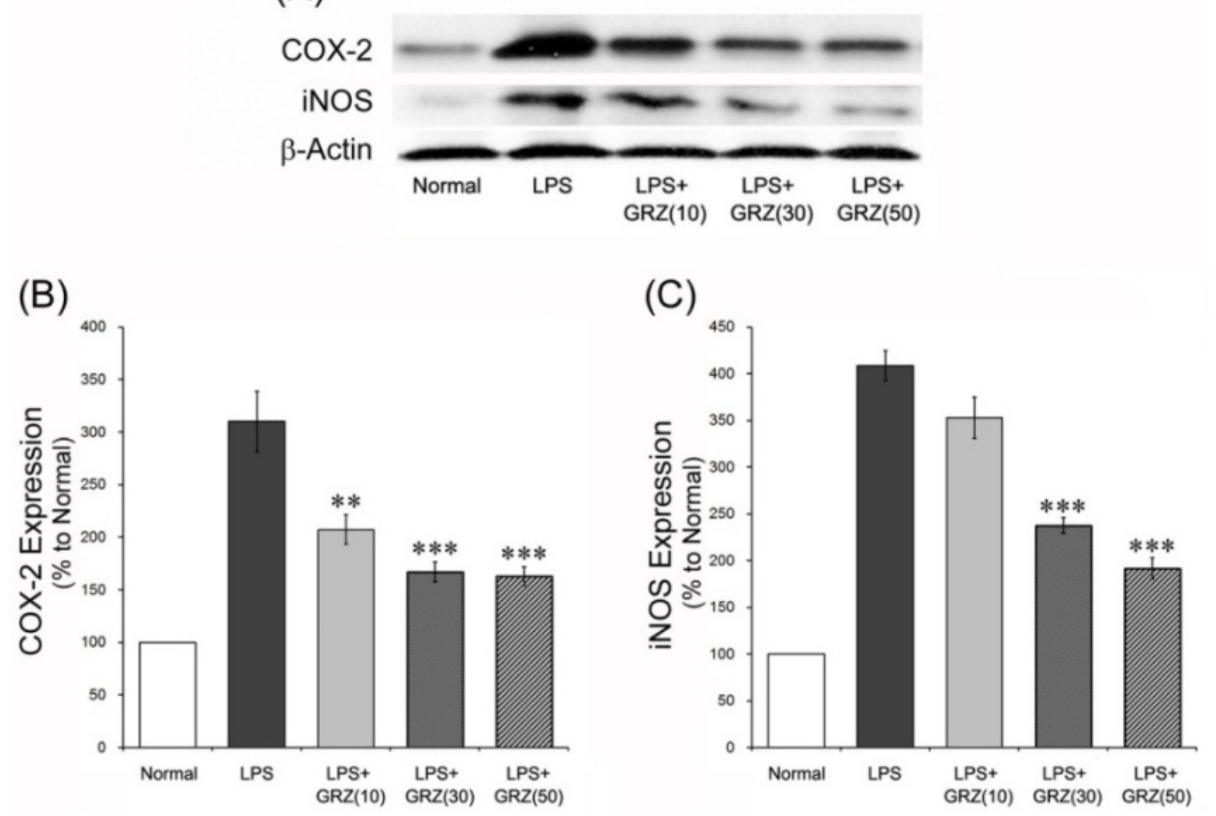

\subsection{Effect on Microglial Activation in the Hippocampal Tissue of LPS-Treated Mice}

To better understand GRZ's anti-neuroinflammatory effect in the hippocampus, observation of microglial activation in the hippocampal tissue of mice was performed in the Morris water maze test. Microglia are key cellular elements of the acute neuroinflammatory response and the primary source for pro-inflammatory cytokines detected in the brain [23]. Systemic LPS injection promotes neuroinflammation through microgial activation and overproduction of inflammatory cytokines [34]. Activated microglia are also responsible for the induction of COX-2 and iNOS in the brain following LPS stimulation [35]. Ionized calcium binding adaptor molecule 1 (Iba1) protein expression, a marker of microglial activation in the hippocampal tissue was measured $24 \mathrm{~h}$ after the LPS injection using Western blotting method. GRZ treatment significantly reduced Ibal expression at a dose of $30 \mathrm{mg} / \mathrm{kg}$ $(p<0.05)$ compared to the LPS group (Figure 4A). When microglia are activated in response to immunological stimulation such as LPS, they undergo morphological changes which include 
shortening and thickening of processes and cell size increase [23]. Therefore, cell number and cell size of Iba1-expressing microglia in the CA1 and DG region of the hippocampus were measured. Although GRZ treatment did not attenuate the number of Iba1-expressing microglia both in the CA1 and DG region of the hippocampus compared to that of the LPS group (Figure 4B,C), the average cell size of the Iba1-expressing microglia was significantly decreased by GRZ treatment both in the CA1 $(p<0.05)$ and DG region $(p<0.05)$ of the hippocampus compared to that of the LPS group (Figure 4B,D). The results indicate that GRZ attenuated the microglial activation related over-expression of pro-inflammatory cytokines in the hippocampus induced by systemic LPS treatment.

Figure 4. Effects of GRZ on Iba1 protein expression and Iba1-expressing microglia in the hippocampal tissue. Representative western blots illustrating differences in the bands of Iba1 (A) GRZ (30 mg/kg) significantly attenuated the up-regulation of hippocampal Ibal expression of mice which performed the Morris water maze test. Representative Iba1-expressing microglia in the hippocampus of mice which performed the Morris water maze test. Scale bars are $100 \mu \mathrm{m}$ (B) The numbers of Iba1-expressing microglia were not different between groups $(\mathbf{C})$. The average cell size of Ibal-expressing microglia was significantly attenuated at $30 \mathrm{mg} / \mathrm{kg}$ of GRZ; and (D) Data are represented by mean $\pm \mathrm{SEM}$ ( $\mathrm{n}=6$ in each group; $\dagger \dagger \uparrow<0.001$ compared to the normal group; $* p<0.05$ compared to the LPS group).

(A)

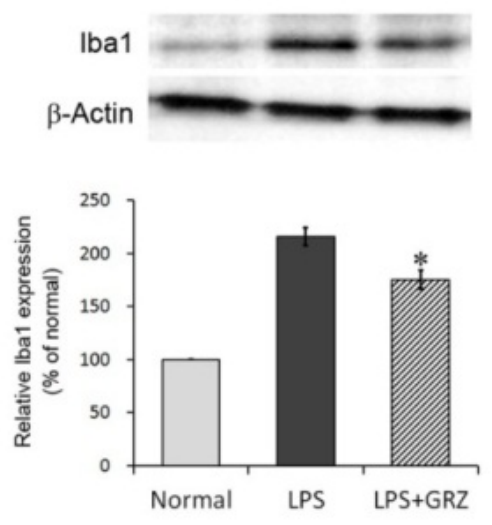

(B) Normal LPS LPS+GRZ

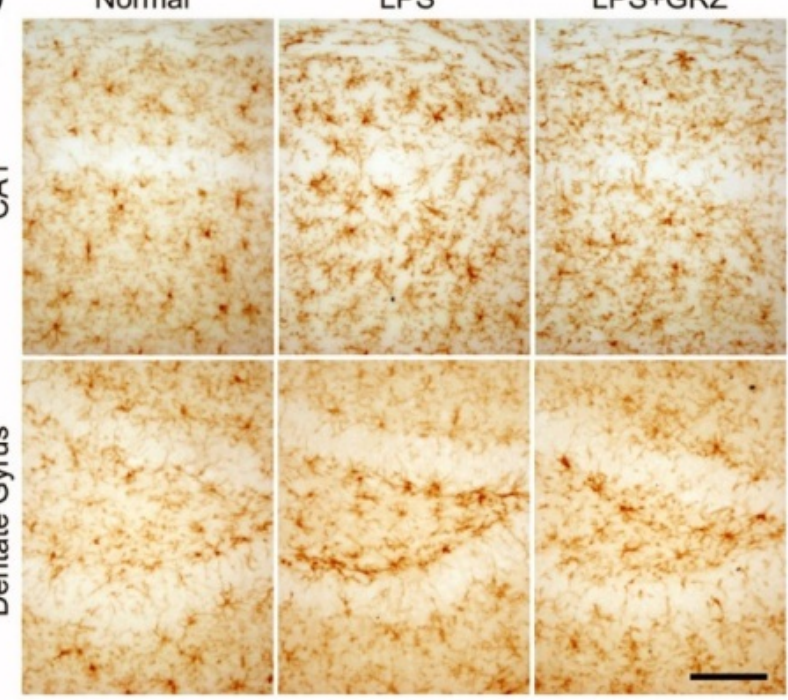

(C)

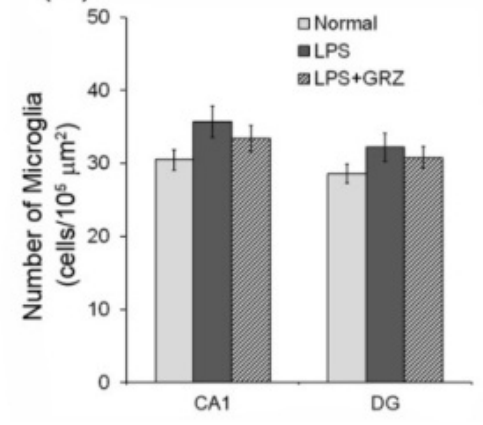

(D)

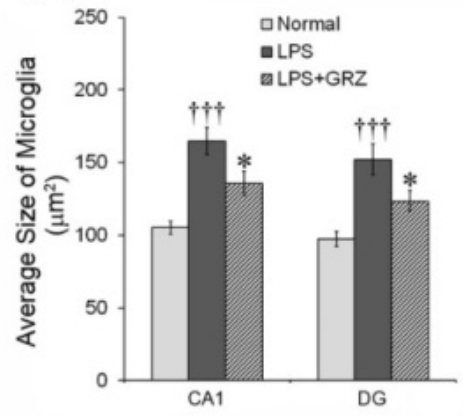




\subsection{Effect on Spatial Learning of LPS-Treated Mice}

TNF- $\alpha$ and IL- $1 \beta$ are the most potent pro-inflammatory cytokines to induce behavioral alterations. Elevated levels of TNF- $\alpha$ have been demonstrated in AD patients [36]. TNF- $\alpha$ protein synthesis inhibitor reversed cognitive deficits induced by chronic LPS-infusion into the ventricle of rats [37]. IL-1 $\beta$ reduced adult hippocampal neurogenesis [38], induced synaptic loss of hippocampal neurons [39], and aggravated long-term potentiation (LTP) and synaptic plasticity in the hippocampus [40]. Direct central administration of IL-1 $\beta$ impaired hippocampal-dependent learning and memory [41]. Moreover, microglial activation is involved in learning and memory impairment through the release of TNF- $\alpha$ and IL-1 $\beta$ and the negative impact on hippocampal LTP [42]. There are numerous reports that systemic LPS treatment produces learning and memory impairment, even if it is acutely stimulated by a single injection of LPS [19]. In the acquisition trials before the LPS treatment, all study groups showed relatively comparable results in the escape latency on the 1 st day $\left(\mathrm{F}_{2,21}=0.255, p=0.777\right)$ and 2 nd day $\left(\mathrm{F}_{2,21}=0.159, p=0.854\right)$. After LPS treatment on the 3rd day, the escape latency of the LPS group was significantly longer compared to the normal group $\left(\mathrm{F}_{1,14}=30.16, p<0.001\right)$, while the escape latency between groups (normal, LPS, and LPS + GRZ) was significantly different $\left(\mathrm{F}_{2,21}=12.19\right.$, $p<0.001)$. The LPS + GRZ group showed significantly shorter escape latency at the 7th and 8th trials on the $3 \mathrm{rd}$ day ( $p<0.05$, respectively), while the escape latencies for all the trials on the $3 \mathrm{rd}$ day were significantly shorter than that of the LPS group $\left(\mathrm{F}_{1,14}=5.164, p<0.05\right)$ (Figure 5). The results indicate that pre-treatment of GRZ was effective in improving spatial learning of LPS-treated mice.

Figure 5. Effect of GRZ on the acquisition training trials. The escape latencies on the 1st and 2nd day were not different among the normal, LPS, and LPS + GRZ groups $\left(\mathrm{F}_{2,21}=0.33\right.$, $p=0.723 ; \mathrm{F}_{2,21}=0.13, p=0.882$; respectively). The LPS + GRZ group showed significantly shorter escape latency at the 7 th and 8 th trials on the 3rd day, while the escape latency for the total trials on the 3rd day was significantly different from the LPS group $\left(\mathrm{F}_{1,14}=1.98, p=0.181\right)$. Data are represented by mean $\pm \mathrm{SEM}(\mathrm{n}=12$ in each group; $\dagger p<0.05 ; \dagger \dagger p<0.01 ; \dagger \dagger p<0.001$ compared to the normal group; ${ }^{*} p<0.05$ compared to the LPS group).

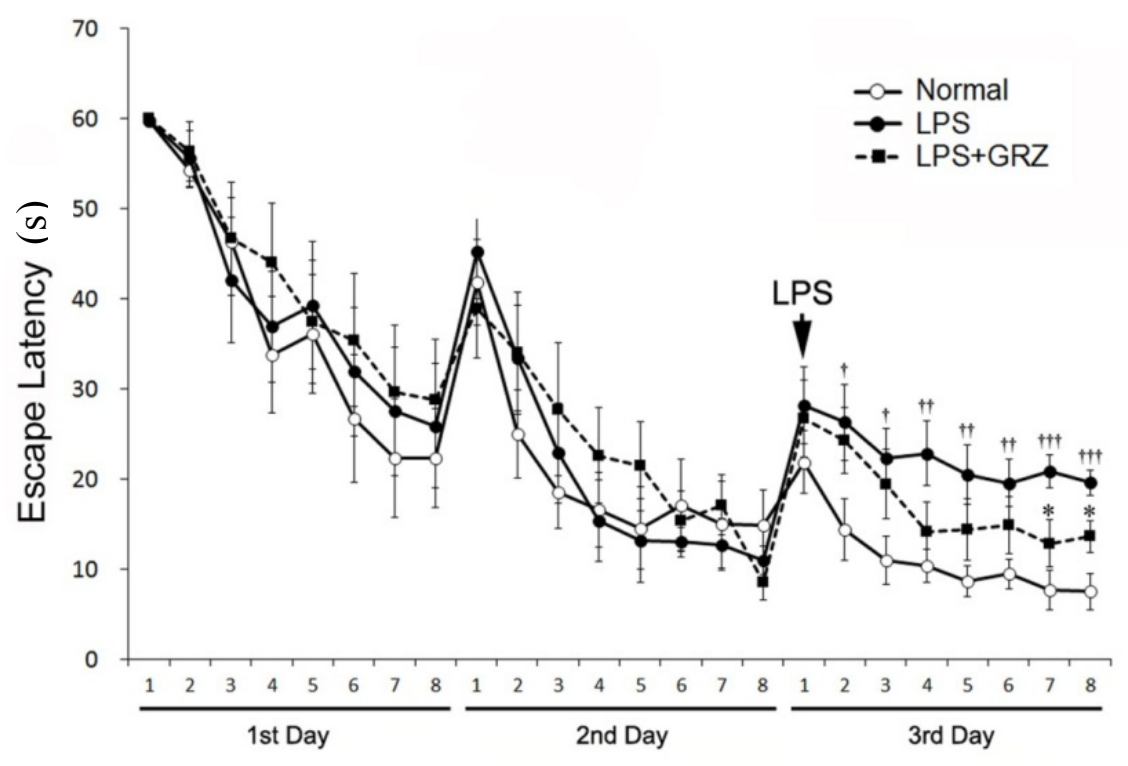




\subsection{Effects on Memory Deficit of LPS-Treated Mice}

In the retention trial on the 4th day of the Morris water maze test, the swimming time spent in the various zones, number of the target heading and memory score were analyzed with a grid design of six zones (Figure 6A) and were used to estimate spatial memory. The LPS group spent significantly less time in zones A (the target, $p<0.001$ ) and B (peri-target area, $p<0.001$ ), but significantly more time in zone $\mathrm{F}$ (dis-target area, $p<0.001$ ) compared to those of the normal group. GRZ treatment significantly prolonged the swimming time spent in zones $\mathrm{A}(p<0.01)$ and $\mathrm{B}(p<0.01)$ and significantly shortened the swimming time spent in zone $\mathrm{F}(p<0.05)$ compared to that of the LPS group (Figure 6B). The number of target heading in the LPS group was significantly reduced $(p<0.01)$ compared to the normal group, while GRZ treatment significantly increased the number of target heading $(p<0.05)$ compared to the LPS group (Figure 7A,B). The LPS group revealed significantly lower memory score $(p<0.001)$ than that of the normal group, while the memory score of the LPS + GRZ group was significantly higher $(p<0.01)$ than that of the LPS group (Figure 7A,C). The results indicate that pre-treatment of GRZ was effective in ameliorating spatial memory of LPS-treated mice. The average swimming speed $(\mathrm{cm} / \mathrm{sec})$ in the retention trials was not different among three groups (normal, $21.9 \pm 1.5$; LPS, $20.2 \pm 0.5$; LPS + GRZ, $21.3 \pm 1.2$ ). The result suggests that GRZ might not affect locomotor and emotional behavior.

Figure 6. Effects of GRZ on the swimming time spent in discrete zones. Computerized grid design used in the retention test. Discrete zones are labeled with letters, zone A representing the platform site (A) GRZ significantly prolonged the swimming time spent in zones A and B, while significantly shortened in zone F; and (B) Data are represented by mean \pm SEM ( $\mathrm{n}=12$ in each group; $\dagger \dagger \dagger p<0.001$ compared to the normal group; ${ }^{*} p<0.05$; ** $p<0.01$ compared to the LPS group).
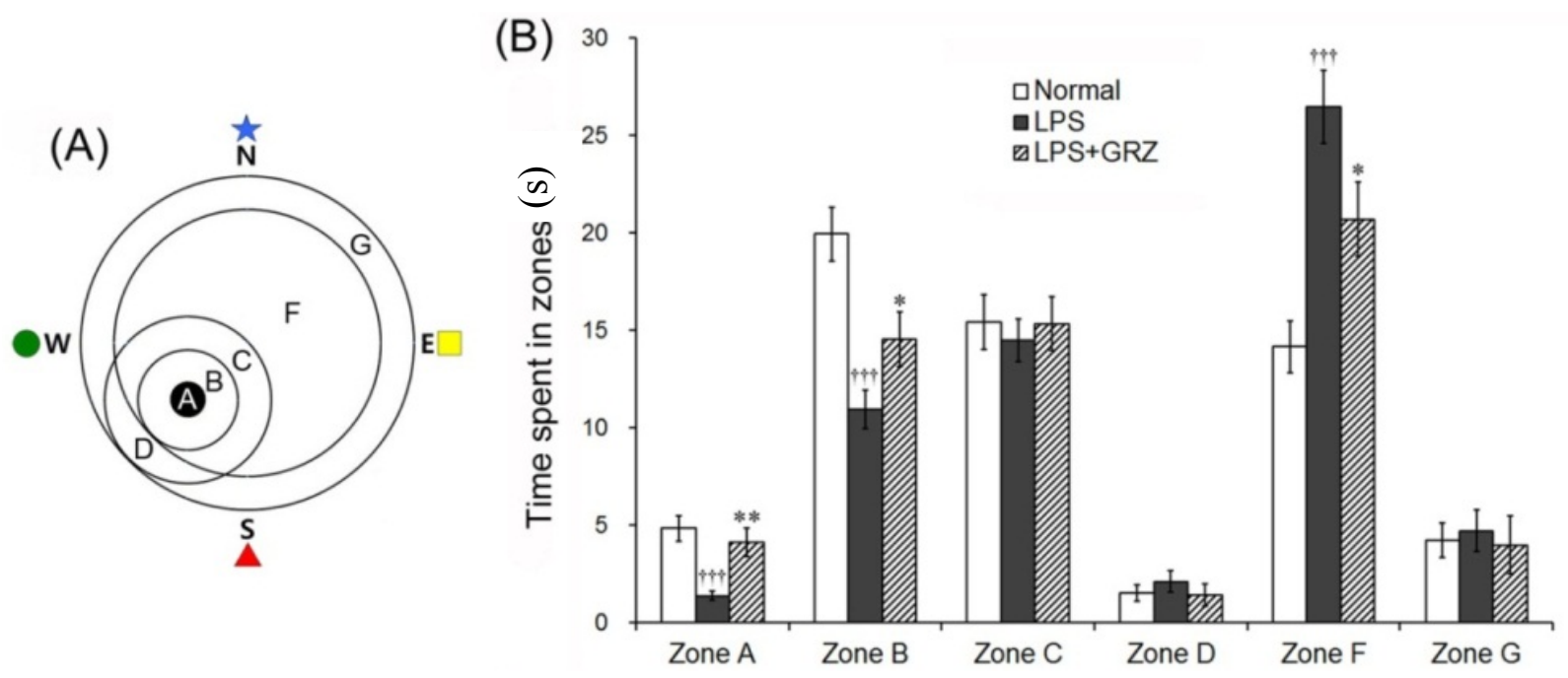
Figure 7. Effects of GRZ on the retention memory test. Representative swimming tracts of the normal, LPS, and LPS+GRZ groups (A) GRZ significantly increased the number of target heading on platform site $(\mathbf{B})$ and memory score; and $(\mathbf{C})$ in the retention test. Data are represented by mean \pm SEM $(n=12$ in each group; $\dagger \dagger p<0.01$; $\dagger \dagger \dagger p<0.001$ compared to the normal group; ${ }^{*} p<0.05 ;{ }^{* *} p<0.01$ compared to the LPS group).

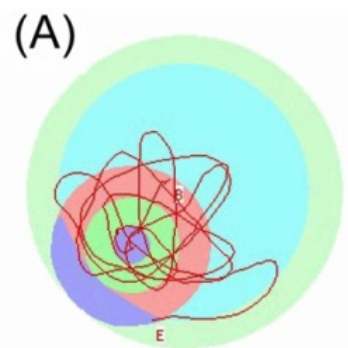

Normal

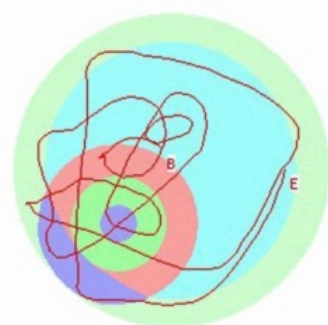

LPS

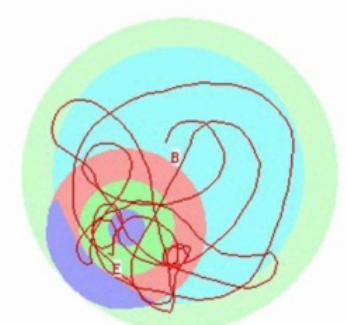

LPS+GRZ
(B)

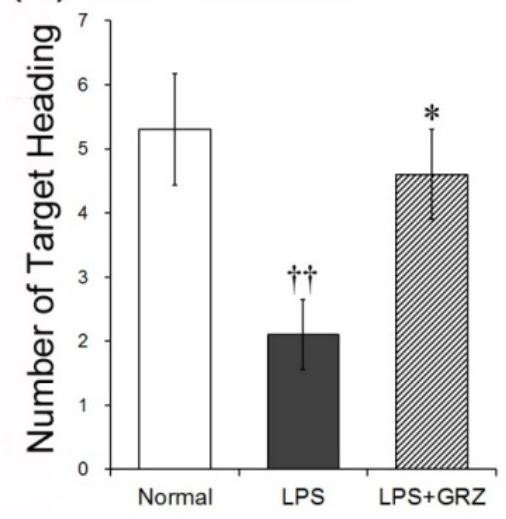

(C)

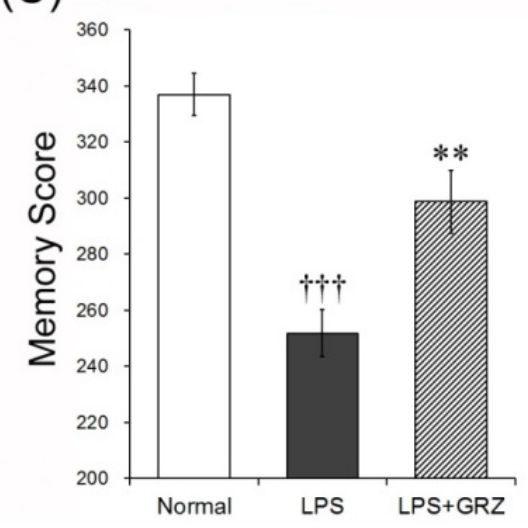

Recently, Zhu et al. and Zhao et al. demonstrated that diammonium glycyrrhizinate, a salt form of GRZ, significantly decreased the escape latency and search distance and increased the target crossing times of $A \beta(1-42)$-induced $A D$ mice [16,17]. The previous reports showed the anti-inflammatory effect of GRZ using in vitro BV-2 cells. This study demonstrated the anti-neuroinflammatory effect of GRZ using the brain tissue in vivo and used a salt form of GRZ, monoammonium glycyrrhizinate, which has more stable and more significant bioactivities than GRZ. TNF- $\alpha$ and IL-1 $\beta$ inhibit LTP in the CA1 and the dentate gyrus regions of the hippocampus [43]. COX-2 inhibition improves suppression of memory and synaptic plasticity [44]. iNOS upregulation interrupts memory consolidation by altering cholinergic function [45]. Therefore, suppression of these neuroinflammatory mediators can lead to improvement in cognitive function. In the present study, GRZ significantly reduced the up-regulations of TNF- $\alpha$, IL- $1 \beta$, COX-2, and iNOS in the brain tissue induced by LPS treatment. Considering all results in this study, it is suggested that GRZ effectively ameliorated the memory deficits induced by systemic LPS treatment through the inhibition of pro-inflammatory cytokines and microglial activation in the brain tissue. 


\section{Experimental}

\subsection{Animals}

Male C57BL/6 mice (25-28 g, Nara Biotechnology, Seoul, Korea) were used for this study. All animal protocols were approved by the Ethics Committee for the Care and Use of Laboratory Animals at Kyung Hee University. The animals were housed in plastic cages at constant temperature $\left(22 \pm 2{ }^{\circ} \mathrm{C}\right)$ and humidity $(55 \pm 10 \%)$ with $12 \mathrm{~h}-12 \mathrm{~h}$ light-dark conditions. The animals were allowed free access to food and water before the experiment.

\subsection{Materials}

Glycyrrhizin (a salt form of GRZ; monoammonium glycyrrhizinate from glycyrrhiza root, $\mathrm{C}_{42} \mathrm{H}_{62} \mathrm{O}_{16} \cdot \mathrm{NH}_{3}$; Figure 8) and lipopolysaccharide (LPS from Escherichia coli O55:B5) were purchased from Sigma-Aldrich (St. Louis, MO, USA). Anti-COX-2 antibody was purchased from Cayman Chemical (Ann Arbor, MI, USA); Anti-iNOS) antibody from BD Biosciences (Laguna Hills, CA, USA); Anti-Iba1 antibodies (\#016-20001, \#019-19741) from Wako Pure Chemical Industries (Osaka, Japan); Anti- $\beta$-actin antibody from Chemicon International (Temecula, CA, USA); Anti-TNF- $\alpha$ antibody from Santa Cruz Biotechnology (Santa Cruz, CA, USA); and Cy2-conjugated donkey antimouse or donkey anti-rabbit IgG from Jackson ImmunoReseach Laboratories (West Grove, PA, USA).

Figure 8. Chemical structure of GRZ (a salt form, monoammonium glycyrrhizinate).

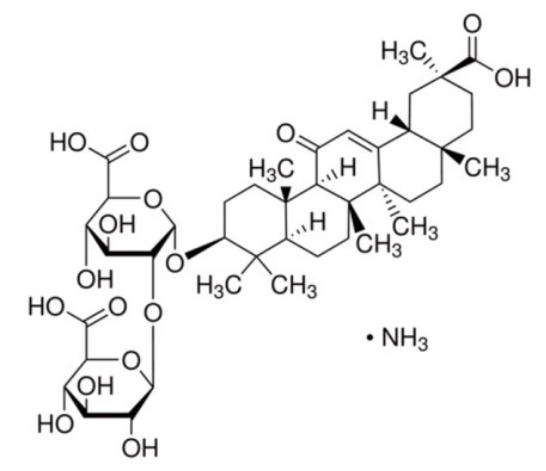

\subsection{Experimental Groups}

For the quantitative real-time polymerase chain reaction (PCR) and western blotting studies, mice were randomly divided into five groups. The normal group (Normal) was allowed free access to food and water without any treatment. The control group (LPS) was intraperitoneally (i.p.) injected with a single dose of LPS (3 mg/kg) and received vehicle (normal saline) orally. The GRZ treatment groups [LPS + GRZ(10), LPS + GRZ(30), LPS + GRZ(50)] were administered GRZ (10, 30, or 50 mg/kg, dissolved in normal saline, orally) respectively, once daily for 3 days before receiving LPS injection. For the Morris water maze study, mice were randomly divided into three groups. The normal group (Normal) was also allowed free access to food and water without any treatment. While the control group (LPS) was intraperitoneally injected with a single dose of LPS (3 mg/kg) on the 3rd day of the experiment. The GRZ treatment group (LPS + GRZ) was administered $30 \mathrm{mg} / \mathrm{kg}$ of GRZ, a reliable dose from the PCR and western blotting studies, once daily for 3 days prior to the LPS injection. They 
were also administered one additional dose $2 \mathrm{~h}$ before the retention test on 4 th day of the experiment. A total of 66 mice, 30 mice for the PCR and the western blotting study and 36 mice for the Morris water maze study were used.

\subsection{Real-Time PCR Measurement}

Pro-inflammatory cytokines, TNF- $\alpha$ and IL-1 $\beta$ in the brain tissue were measured using real-time PCR method. Twenty-four hours after the LPS injection, the mice were sacrificed by decapitation and the brain was rapidly dissected on ice. Total RNA was extracted from the samples with Trizol (Qiagen, Germany) according to the manufacturer's protocol. One microgram of total RNA was transcribed into DNA using iScript cDNA synthesis Kit (Bio-Rad, Hercules, CA, USA). After reverse transcription, quantitative real-time PCR was performed using preoptimized primer/probe mixture with iQ SYBR Green Supermix kit (Bio-Rad) and the CFX 96 RT-PCR Detection System (Bio-Rad). Primer sequences for the analyzed genes were as follows: (1) TNF- $\alpha$; forward, 5'-TGA GAA GTT CCC AAA TGG C-3'; reverse, 5'-GCT ACA GGC TTG TCA CTC-3'; (2) IL-1 $\beta$; forward, 5'-TGA GCA CCT TCT TTT CCT TCA-3'; reverse, 5'-TTG TCT AAT GGG AAC GTC ACA C-3'; (3) $\beta$-actin; forward, 5'-TTT CCA GCC TTC CTT GGG TAT G-3'; reverse, 5'-CAC TGT GTT GGC ATA GAG GTC TTT AC-3'. The relative difference in expression between samples is represented by cycle time values normalized to the measurement of the housekeeping gene $\beta$-actin as a reference. The sample values represent $\mathrm{x}$-fold differences from a normal sample (given as a designated value of 1) within the same experiment.

\subsection{Western Blotting}

COX-2 and iNOS expression in the brain tissue and Ibal expression in the hippocampal tissue were measured by Western blotting method. The brain tissue was homogenized and sonicated on ice in lysis buffer $(50 \mathrm{mM}$ Tris- $\mathrm{HCl}$, $\mathrm{pH}$ 8.0, $150 \mathrm{mM} \mathrm{NaCl}, 1 \%$ Triton X-100, 0.5\% sodium deoxycholate, $0.1 \%$ sodium dodecyl sulfate (SDS), $1 \mathrm{mM}$ EDTA, $1 \%$ protease inhibitor cocktail; Sigma). After centrifugation, the supernatant was collected and assayed for protein concentration using the Bradford method. Lysate samples containing $50 \mu \mathrm{g}$ of protein were fractionated by SDS $-10 \%$ polyacrylamide gel electrophoresis, and then subjected to western blot analysis. The primary antibodies used in this study were mouse anti-COX-2 antibody (\#160106, Cayman), mouse anti-iNOS antibody (\#610329, BD Biosciences), rabbit anti-Iba1 antibody (\#016-20001, Wako) and mouse anti- $\beta$-actin antibody (Chemicon). Ibal expression in the hippocampal tissue was examined in the mice which performed the Morris water maze test.

\subsection{Morris Water Maze Test}

The Morris water maze test was performed for 4 days. The acquisition training was performed for 3 days prior to the LPS injection and the retention test on the 4th day. The apparatus consisted of a circular water pool $100 \mathrm{~cm}$ in diameter and $40 \mathrm{~cm}$ in height. It was filled with $23 \pm 1{ }^{\circ} \mathrm{C}$ water with a depth of $28 \mathrm{~cm}$ and covered a black platform $(10 \mathrm{~cm}$ in diameter). The platform was submerged approximately $0.5 \mathrm{~cm}$ below the surface of water. The pool was divided into four quadrants: northeast 
(NE), northwest (NW), southeast (SE), and southwest (SW) at equal distances on the rim. The platform was located in the center of the southwest quadrant. During the first 3 days of acquisition tests, mice were given eight trials per day to find the hidden platform. Each mouse (12 mice per group) was gently placed into the water facing the wall in the direction of north $(\mathrm{N})$, east $(\mathrm{E})$, south $(\mathrm{S})$, and west (W) in two series of order. The mouse was allowed to swim until they reached the hidden platform (maximum swim time was $60 \mathrm{~s}$ ). The escape latency to reach the platform was recorded and they remained on the platform for $20 \mathrm{~s}$ before being removed. The mouse which failed to find the platform within $60 \mathrm{~s}$ was guided to the hidden platform and then was placed on the platform for $20 \mathrm{~s}$ for reinforcement before being removed. On the 3rd day, LPS was injected into the mice $1 \mathrm{~h}$ before the acquisition test.

One trial of the retention test without the platform was performed on the 4 th day, $24 \mathrm{~h}$ after the LPS injection, to assess the memory of the correct platform location. The mice were placed into the pool and swam freely for $60 \mathrm{~s}$. The swimming paths were recorded by a video camera linked to a computerbased image analyzer (SMART 2.5 video-tracking system, Panlab, Barcelona, Spain). The number of target heading and the swimming time in each zone was analyzed with a grid design of 6 zones (Figure 6A). This grid design, constructed with a computer-based image analyzer, was superimposed over the maze and viewed on a monitor. Memory scores were calculated using the formula (time in zone $\mathrm{A} \times 10)+($ time in zone $\mathrm{B} \times 8)+($ time in zone $\mathrm{C} \times 6)+($ time in zone $\mathrm{D} \times 3)+($ time in zone $\mathrm{F} \times 2)+($ time in zone $\mathrm{G} \times 1)=$ memory score. The grid design and the formula for calculating the memory score were based on and modified from the behavior study of Smith et al. [46]. The mice were sacrificed after the retention test trial and the brains were randomly used either for Ibal western blotting (6 mice) or immunohistochemistry (6 mice).

\subsection{Immunohistochemistry}

After the retention test trail, the mice were sacrificed and the brains (6 mice per group, randomly) were used for immunohistochemistry against TNF- $\alpha$, COX-2, and iNOS expression in the brain tissue. The mice were deeply anesthetized and perfused transcardially with $0.05 \mathrm{M}$ phosphate-buffered saline (PBS) containing 4\% paraformaldehyde. The brain was removed and was postfixed in the same perfusing solution overnight at $4{ }^{\circ} \mathrm{C}$. Thirty $\mu \mathrm{m}$ thick coronal sections of brain tissue were made using a freezing microtome (Leica, Wetzler, Germany). The brain sections were rinsed with $0.05 \mathrm{M}$ PBS and incubated for $15 \mathrm{~min}$ in $1 \%$ hydrogen peroxide PBS at room temperature. The sections were incubated overnight at $4{ }^{\circ} \mathrm{C}$ with primary antibodies against TNF- $\alpha$ (1:200, sc-1349, Santa Cruz), COX-2 (1:200, \#160106, Cayman), and iNOS (1:200, \#610329, BD), then incubated with anti-rabbit or anti-mouse Cy2 as a secondary antibody (Jackson ImmunoResearch, West Grove, PA, USA). The fluorescencelabeled images were captured using a confocal laser-scanning microscope (Carl Zeiss, LSM 510 META, Oberkochen, Germany). The fluorescence-labeled densities against TNF- $\alpha$, COX-2, and iNOS were analyzed using ImageJ software (Ver. 1.44p, NIH, Bethesda, MD, USA) in corresponding areas. The sample values represent the percentage increase differences from a sample of the normal group and the mean values for the four sections in each mouse were used for statistical analysis. For Iba1 immunohistochemistry, Iba1 (1:500, \#019-19741, Wako) was used for primary antibody and the avidin-biotin complex (Vector Laboratories, Burlingame, CA, USA) method were carried out with peroxidase coupling in a mixture containing $0.05 \%$ diaminobenzidine (Sigma-Aldrich) and $0.03 \%$ 
$\mathrm{H} 2 \mathrm{O} 2$ for 2-5 min. The Iba1-expressing microglia were captured using a light microscope (BX51, Olympus, Tokyo, Japan) equipped with CCD camera (DP70, Olympus) and analyzed using the ImageJ software (Ver. $1.44 \mathrm{p}, \mathrm{NIH}, \mathrm{USA})$. The number and the average size of microglia in the CA1 and DG region of the hippocampus were measured on an inverted black-white binary image by determination of threshold gray value and pixels definition using the ImageJ software. Data were normalized with the same area $(105 \mu \mathrm{m} 2)$ and the mean values from the four sections analyzed in each mouse were used for statistical analysis.

\subsection{Statistical Analysis}

Study data are presented as means \pm standard errors. Differences between groups were evaluated using paired Student's t-test and one-way Analysis of variance (ANOVA). A probability value of less than 0.05 was used to indicate a significant difference.

\section{Conclusions}

This study demonstrates that GRZ effectively reduced neuroinflammation and ameliorated the memory deficits induced by systemic LPS treatment. The effects of GRZ were found to be mediated through the inhibition of pro-inflammatory cytokines and microglial activation in the brain tissue. Therefore, this study supports that GRZ may be a putative therapeutic drug on neurodegenerative diseases associated with cognitive deficits and neuroinflammation such as AD.

\section{Acknowledgments}

We thank to Dong-Hui Lee and Myeong-Han Ryu (college students in the Department of Medical Science, Kyung Hee University) for their diligent participation in the Morris water maze test.

\section{Conflicts of Interest}

The authors declare no conflict of interest.

\section{References}

1. Sharifzadeh, M.; Shamsa, F.; Shiran, S.; Karimfar, M.H.; Miri, A.H.; Jalalizadeh, H.; Gholizadeh, S.; Salar, F.; Tabrizian, K. A time course analysis of systemic administration of aqueous licorice extract on spatial memory retention in rats. Planta Med. 2008, 74, 485-490.

2. Li, X.L.; Zhou, A.G. Evaluation of the immunity activity of glycyrrhizin in AR mice. Molecules 2012, 17, 716-727.

3. Tabuchi, M.; Imamura, S.; Kawakami, Z.; Ikarashi, Y.; Kase, Y. The blood-brain barrier permeability of 18beta-glycyrrhetinic acid, a major metabolite of glycyrrhizin in glycyrrhiza root, a constituent of the traditional japanese medicine yokukansan. Cell. Mol. Neurobiol. 2012, 32, 1139-1146.

4. Wang, C.Y.; Kao, T.C.; Lo, W.H.; Yen, G.C. Glycyrrhizic acid and 18beta-glycyrrhetinic acid modulate lipopolysaccharide-induced inflammatory response by suppression of nf-kappab through pi3k p110delta and p110gamma inhibitions. J. Agric. Food Chem. 2011, 59, 7726-7733. 
5. Feng, L.; Zhu, M.M.; Zhang, M.H.; Wang, R.S.; Tan, X.B.; Song, J.; Ding, S.M.; Jia, X.B.; $\mathrm{Hu}, \mathrm{S} . Y$. Protection of glycyrrhizic acid against ages-induced endothelial dysfunction through inhibiting rage/nf-kappab pathway activation in human umbilical vein endothelial cells. J. Ethnopharmacol. 2013, 148, 27-36.

6. Ni, Y.F.; Kuai, J.K.; Lu, Z.F.; Yang, G.D.; Fu, H.Y.; Wang, J.; Tian, F.; Yan, X.L.; Zhao, Y.C.; Wang, Y.J.; et al. Glycyrrhizin treatment is associated with attenuation of lipopolysaccharideinduced acute lung injury by inhibiting cyclooxygenase- 2 and inducible nitric oxide synthase expression. J. Surg. Res. 2011, 165, e29-e35.

7. Bhattacharjee, S.; Bhattacharjee, A.; Majumder, S.; Majumdar, S.B.; Majumdar, S. Glycyrrhizic acid suppresses cox-2-mediated anti-inflammatory responses during leishmania donovani infection. J. Antimicrob. Chemoth. 2012, 67, 1905-1914.

8. Cherng, J.M.; Lin, H.J.; Hung, M.S.; Lin, Y.R.; Chan, M.H.; Lin, J.C. Inhibition of nuclear factor kappab is associated with neuroprotective effects of glycyrrhizic acid on glutamate-induced excitotoxicity in primary neurons. Eur. J. Pharmacol. 2006, 547, 10-21.

9. Kao, T.C.; Shyu, M.H.; Yen, G.C. Neuroprotective Effects of glycyrrhizic acid and 18beta-glycyrrhetinic acid in pc12 cells via modulation of the pi3k/akt pathway. J. Agric. Food Chem. 2009, 57, 754-761.

10. Kim, S.W.; Jin, Y.; Shin, J.H.; Kim, I.D.; Lee, H.K.; Park, S.; Han, P.L.; Lee, J.K. Glycyrrhizic acid affords robust neuroprotection in the postischemic brain via anti-inflammatory effect by inhibiting hmgb1 phosphorylation and secretion. Neurobiol. Dis. 2012, 46, 147-156.

11. Ohnishi, M.; Katsuki, H.; Fukutomi, C.; Takahashi, M.; Motomura, M.; Fukunaga, M.; Matsuoka, Y.; Isohama, Y.; Izumi, Y.; Kume, T.; et al. HMGB1 inhibitor glycyrrhizin attenuates intracerebral hemorrhage-induced injury in rats. Neuropharmacology 2011, 61, 975-980.

12. Ni, B.; Cao, Z.; Liu, Y. Glycyrrhizin protects spinal cord and reduces inflammation in spinal cord ischemia-reperfusion injury. Int. J. Neurosci. 2013, 123, 745-751.

13. Gong, G.; Yuan, L.B.; Hu, L.; Wu, W.; Yin, L.; Hou, J.L.; Liu, Y.H.; Zhou, L.S. Glycyrrhizin attenuates rat ischemic spinal cord injury by suppressing inflammatory cytokines and hmgb1. Acta Pharmacol. Sin. 2012, 33, 11-18.

14. Kim, J.B.; Sig Choi, J.; Yu, Y.M.; Nam, K.; Piao, C.S.; Kim, S.W.; Lee, M.H.; Han, P.L.; Park, J.S.; Lee, J.K. HMGB1, a Novel cytokine-like mediator linking acute neuronal death and delayed neuroinflammation in the postischemic brain. J. Neurosci. 2006, 26, 6413-6421.

15. Fang, P.; Schachner, M.; Shen, Y.Q. HMGB1 in development and diseases of the central nervous system. Mol. Neurobiol. 2012, 45, 499-506.

16. Zhu, X.; Chen, C.; Ye, D.; Guan, D.; Ye, L.; Jin, J.; Zhao, H.; Chen, Y.; Wang, Z.; Wang, X.; et al. Diammonium glycyrrhizinate upregulates pgc-1alpha and protects against abeta $1-42$-induced neurotoxicity. PLoS One 2012, 7, e35823.

17. Zhao, H.; Wang, S.L.; Qian, L.; Jin, J.L.; Li, H.; Xu, Y.; Zhu, X.L. Diammonium glycyrrhizinate attenuates abeta $_{1-42}$-induced neuroinflammation and regulates mapk and nf-kappab pathways in vitro and in vivo. CNS Neurosci. Ther. 2013, 19, 117-124.

18. McGeer, E.G.; McGeer, P.L. Inflammatory processes in alzheimer's disease. Prog. Neuropsychopharmacol. Biol. Psychiatry 2003, 27, 741-749. 
19. Shaw, K.N.; Commins, S.; O’Mara, S.M. Lipopolysaccharide causes deficits in spatial learning in the watermaze but not in bdnf expression in the rat dentate gyrus. Behav. Brain Res. 2001, $124,47-54$.

20. Lee, J.W.; Lee, Y.K.; Yuk, D.Y.; Choi, D.Y.; Ban, S.B.; Oh, K.W.; Hong, J.T. Neuro-Inflammation induced by lipopolysaccharide causes cognitive impairment through enhancement of Beta-Amyloid generation. J. Neuroinflamm. 2008, 5, 37.

21. Lien, E.; Means, T.K.; Heine, H.; Yoshimura, A.; Kusumoto, S.; Fukase, K.; Fenton, M.J.; Oikawa, M.; Qureshi, N.; Monks, B.; et al. Toll-Like receptor 4 imparts ligand-specific recognition of bacterial lipopolysaccharide. J. Clin. Invest. 2000, 105, 497-504.

22. Block, M.L.; Zecca, L.; Hong, J.S. Microglia-Mediated neurotoxicity: Uncovering the molecular mechanisms. Nat. Rev. Neurosci. 2007, 8, 57-69.

23. Graeber, M.B.; Streit, W.J. Microglia: Biology and pathology. Acta Neuropathol. 2010, 119, 89-105.

24. Liu, X.; Wu, Z.; Hayashi, Y.; Nakanishi, H. Age-Dependent neuroinflammatory responses and deficits in long-term potentiation in the hippocampus during systemic inflammation. Neuroscience 2012, 216, 133-142.

25. Thomson, L.M.; Sutherland, R.J. Systemic administration of lipopolysaccharide and interleukin1 beta have different effects on memory consolidation. Brain Res. Bull. 2005, 67, 24-29.

26. Dantzer, R.; Bluthe, R.M.; Gheusi, G.; Cremona, S.; Laye, S.; Parnet, P.; Kelley, K.W. Molecular basis of sickness behavior. Ann. N. Y. Acad. Sci. 1998, 856, 132-138.

27. Hennigan, A.; Trotter, C.; Kelly, A.M. Lipopolysaccharide impairs long-term potentiation and recognition memory and increases p75ntr expression in the rat dentate gyrus. Brain Res. 2007, 1130, 158-166.

28. Erickson, M.A.; Banks, W.A. Cytokine and chemokine responses in serum and brain after single and repeated injections of lipopolysaccharide: Multiplex quantification with path analysis. Brain Behav. Immun. 2011, 25, 1637-1648.

29. Jeong, H.K.; Jou, I.; Joe, E.H. Systemic LPS administration induces brain inflammation but not dopaminergic neuronal death in the substantia nigra. Exp. Mol. Med. 2010, 42, 823-832.

30. Park, S.M.; Choi, M.S.; Sohn, N.W.; Shin, J.W. Ginsenoside Rg3 attenuates microglia activation following systemic lipopolysaccharide treatment in mice. Biol. Pharm. Bull. 2012, 35, 1546-1552.

31. Kudo, T.; Okamura, S.; Zhang, Y.; Masuo, T.; Mori, M. Topical application of glycyrrhizin preparation ameliorates experimentally induced colitis in rats. World J. Gastroentero. 2011, 17, 2223-2228.

32. Aid, S.; Bosetti, F. Targeting cyclooxygenases-1 and -2 in neuroinflammation: Therapeutic implications. Biochimie 2011, 93, 46-51.

33. Possel, H.; Noack, H.; Putzke, J.; Wolf, G.; Sies, H. Selective upregulation of inducible nitric oxide synthase (inos) by lipopolysaccharide (lps) and cytokines in microglia: In vitro and in vivo studies. Glia 2000, 32, 51-59.

34. Qin, L.; Wu, X.; Block, M.L.; Liu, Y.; Breese, G.R.; Hong, J.S.; Knapp, D.J.; Crews, F.T. Systemic LPS causes chronic neuroinflammation and progressive neurodegeneration. Glia 2007, $55,453-462$.

35. Sheng, W.; Zong, Y.; Mohammad, A.; Ajit, D.; Cui, J.; Han, D.; Hamilton, J.L.; Simonyi, A.; Sun, A.Y.; Gu, Z.; et al. Pro-Inflammatory cytokines and lipopolysaccharide induce changes in 
cell morphology, and upregulation of ERK1/2, iNOS and SPLA(2)-IIA expression in astrocytes and microglia. J. Neuroinflamm. 2011, 8, 121.

36. Alvarez, A.; Cacabelos, R.; Sanpedro, C.; Garcia-Fantini, M.; Aleixandre, M. Serum TNF-Alpha levels are increased and correlate negatively with free igf-i in alzheimer disease. Neurobiol. Aging 2007, 28, 533-536.

37. Belarbi, K.; Jopson, T.; Tweedie, D.; Arellano, C.; Luo, W.; Greig, N.H.; Rosi, S. TNF-Alpha protein synthesis inhibitor restores neuronal function and reverses cognitive deficits induced by chronic neuroinflammation. J. Neuroinflamm. 2011, doi:10.1186/1742-2094-8-121.

38. Wu, M.D.; Montgomery, S.L.; Rivera-Escalera, F.; Olschowka, J.A.; O’Banion, M.K. Sustained IL-1beta expression impairs adult hippocampal neurogenesis independent of il-1 signaling in nestin+ neural precursor cells. Brain Behav. Immun. 2013, 32, 9-18.

39. Mishra, A.; Kim, H.J.; Shin, A.H.; Thayer, S.A. Synapse loss induced by interleukin-1beta requires pre- and post-synaptic mechanisms. J. Neuroimmune Pharm. 2012, 7, 571-578.

40. Imamura, Y.; Wang, H.; Matsumoto, N.; Muroya, T.; Shimazaki, J.; Ogura, H.; Shimazu, T. Interleukin-1beta causes long-term potentiation deficiency in a mouse model of septic encephalopathy. Neuroscience 2011, 187, 63-69.

41. Goshen, I.; Kreisel, T.; Ounallah-Saad, H.; Renbaum, P.; Zalzstein, Y.; Ben-Hur, T.; Levy-Lahad, E.; Yirmiya, R. A Dual role for interleukin-1 in hippocampal-dependent memory processes. Psychoneuroendocrinology 2007, 32, 1106-1115.

42. Liu, M.C.; Liu, X.Q.; Wang, W.; Shen, X.F.; Che, H.L.; Guo, Y.Y.; Zhao, M.G.; Chen, J.Y.; Luo, W.J. Involvement of microglia activation in the lead induced long-term potentiation impairment. PLoS One 2012, 7, e43924.

43. Cunningham, A.J.; Murray, C.A.; O’Neill, L.A.; Lynch, M.A.; O’Connor, J.J. Interleukin-1 beta (il-1 beta) and tumour necrosis factor (tnf) inhibit long-term potentiation in the rat dentate gyrus in vitro. Neurosci. Lett. 1996, 203, 17-20.

44. Kotilinek, L.A.; Westerman, M.A.; Wang, Q.; Panizzon, K.; Lim, G.P.; Simonyi, A.; Lesne, S.; Falinska, A.; Younkin, L.H.; Younkin, S.G.; et al. Cyclooxygenase-2 inhibition improves amyloid-beta-mediated suppression of memory and synaptic plasticity. Brain 2008, 131, 651-664.

45. Udayabanu, M.; Kumaran, D.; Nair, R.U.; Srinivas, P.; Bhagat, N.; Aneja, R.; Katyal, A. Nitric oxide associated with inos expression inhibits acetylcholinesterase activity and induces memory impairment during acute hypobaric hypoxia. Brain Res. 2008, 1230, 138-149.

46. Smith, D.H.; Okiyama, K.; Thomas, M.J.; McIntosh, T.K. Effects of the excitatory amino acid receptor antagonists kynurenate and indole-2-carboxylic acid on behavioral and neurochemical outcome following experimental brain injury. J. Neurosci. 1993, 13, 5383-5392.

Sample Availability: Not available.

(C) 2013 by the authors; licensee MDPI, Basel, Switzerland. This article is an open access article distributed under the terms and conditions of the Creative Commons Attribution license (http://creativecommons.org/licenses/by/3.0/). 